\title{
Oligodendroglioma: clinical study and survival analysis correlated with chromosomal anomalies
}

\author{
Abderrahmane Hamlat, M.D., Stephan Saikali, M.D., Jacques Chaperon, M.D., \\ Michèle Le Calve, M.D., Daniel Gedouin, M.D., Mohamed Ben-Hassel, M.D., \\ AND Yvon GuEgan, M.D.
}

Departments of Neurosurgery, Pathology, Public Health, and Cytogenetic and Molecular Biology, CHU Pontchaillou; and Departments of Medical Oncology and Radiotherapy, Centre Eugene Marquis, Rennes, France

\begin{abstract}
Object. Demonstration of the loss of chromosomes $1 \mathrm{p}$ and $19 \mathrm{q}$ in the presence of a brain neoplasm marks the emergence of genotype as a prognostic indicator. The authors report gene expression data for oligodendroglioma and correlate genotype with response to therapy. Gene expression subgroups may represent distinct types of disease.

Methods. Eighty-seven cases of supratentorial oligodendroglioma were selected from 145 cases treated in a single center between January 1990 and December 2001. Fluorescence in situ hybridization was used to determine the status of chromosomes $1 \mathrm{p}$ and 19q. Parameters evaluated included clinical data and radiological and histological features. Univariate and multivariate analyses were performed and a probability value less than 0.05 was considered significant.

The patients included 48 women and 39 men. The overall mean age at presentation was 45 years for women and 36 years for men $(\mathrm{p}=0.006)$. The univariate analysis identified the following as favorable prognostic factors: younger patient age $\left(\mathrm{p}=10^{-5}\right)$, female sex $(\mathrm{p}=0.0025)$, seizure as a presenting symptom $\left(\mathrm{p}=10^{-5}\right)$, normal clinical examination $\left(\mathrm{p}=10^{-5}\right)$, absence of lesion enhancement on neuroimaging studies $(\mathrm{p}=0.0231)$, lack of histological necrosis $(\mathrm{p}=$ $0.0003)$, absence of mitoses $(p=0.0014), 1 p$ and $19 q$ deletions $(p=0.0001)$, absence of recurrence $(p=0.0021)$, and adjuvant radiotherapy and/or chemotherapy $\left(\mathrm{p}=10^{-5}\right)$. The multivariate analysis identified patient age $\left(\mathrm{p}=10^{-5}\right)$ and chromosomal anomalies $(\mathrm{p}=0.002)$ as independently linked to survival. Three molecular subtypes emerged: oligodendroglioma with $1 \mathrm{p}$ and $19 \mathrm{q}$ deletions, oligodendroglioma demonstrating polysomia and a lack of meaningful response to radiotherapy or chemotherapy, and oligodendroglioma with no $1 \mathrm{p}-9 \mathrm{q}$ deletion in which partial response was seen.

Conclusions. According to our data, oligodendrogliomas could be divided into three molecular subtypes. Although chemotherapy seems efficient for managing this tumor, additional studies should be conducted to compare the efficacy of radiotherapy and chemotherapy.
\end{abstract}

\section{KEY WORDS • oligodendroglioma $•$ fluorescence in situ hybridization $・$ 1p-19q deletion • polysomia - prognosis}

Recent studies have shown that the combined loss of chromosomes $1 \mathrm{p}$ and $19 \mathrm{q}$ is a genetic predictor of chemosensitivity and prolonged overall survival in oligodendroglioma. ${ }^{5,6,40,44}$ Fifty to seventy percent of oligodendroglial tumors are characterized by a loss of the short arm of chromosome $1(1 \mathrm{p})$ and the long arm of chromosome 19 $(19 q), 3,5,6,15,34,40,44$ and practically all tumors with this combined loss of $1 \mathrm{p}$ and $19 \mathrm{q}$ respond to chemotherapy. ${ }^{6,15}$ The demonstration of this correlation made genotyping of brain tumors a useful indicator of therapeutic outcome. ${ }^{6,44}$

Chromosomal studies are performed using several techniques $^{3,17,18,31,34,40}$ including FISH. Fluorescence in situ hybridization is a molecular cytogenetic investigation that uses recombinant DNA technology to analyze both metaphase and interphase cells. Because it can assess both di-

Abbreviations used in this paper: $\mathrm{CI}=$ confidence interval; $\mathrm{CT}=$ computerized tomography; FISH = fluorescence in situ hybridization; $\mathrm{MR}=$ magnetic resonance; $\mathrm{WHO}=$ World Health Organization. viding and nondividing cells, FISH can make an important contribution to retrospective studies. . $9,29,34^{2}$

The possibility that gene expression subgroups may represent distinct types of a disease ${ }^{2,6,12-14,42}$ has led us to describe gene expression data for oligodendroglioma in relation to its biological behavior and its response to therapy.

\section{CLINICAL MATERIAL AND METHODS}

The surgical pathology files of the neurosurgery department of Rennes University Hospital were searched for cases of oligodendroglioma. Records of patients who presented between January 1990 and December 2001 were selected. All patients were initially admitted to the neurosurgery department for surgical procedures and histological diagnosis.

\section{Histopathological Analysis}

All initial histological specimens were reviewed for confirmation of the original diagnosis by a single neuropathol- 
ogist who was blinded to patient data except for presence or absence of contrast enhancement.

The definition of oligodendroglioma was that according to the WHO. ${ }^{15}$ The histological parameters that were evaluated included necrosis, vascular proliferation, mitosis, and cell density. Cellularity was defined as low, moderate, or high according to the presence or absence of neuropil between tumor cells. Necrosis was recorded as present when observed in at least one area in the total histological samples examined. Mitosis counts were performed in the most mitotically active area of the tumor at high-power magnification. The single highest mitosis count per $10 \mathrm{hpf}$ was recorded. Endothelial proliferation was defined as low rate when confluence between endothelial cells was observed and as high rate when glomeruloid aspects were observed.

All tumors were classified according to both $\mathrm{WHO}^{20}$ (Grades II and III) and St. Anne (Grades A and B) classifications. ${ }^{9,10}$ Tumors were classified as low grade (A-II) when two criteria were present. These included no or few mitoses $(<5 / \mathrm{hpf})$, low or moderate cellular density, and/or a low vascular proliferation rate. Specimens were classified as high grade (B-III) if they showed high cellular density, intense vascular proliferation, numerous mitoses, and necrosis (and contrast enhancement for the St. Anne grading system).

Immunohistochemical studies involving glial fibrillary acidic protein, neurofilament, and Ki-67 stains were performed on all tumors. The presence of vascular endothelial growth factor, p27, p18, and MIB1 were retrospectively assessed in 84 specimens. Glial fibrillary acidic protein staining was always negative except in astrocytic areas $(<20 \%$ of the total sample examined). Staining for neurofilament was negative and that for Ki-67 was usually positive.

\section{Fluorescence in Situ Hybridization Analysis}

Fluoresence in situ hybridization was used to evaluate allelic losses of chromosomes 1p36 and 19q13 on formalinfixed paraffin-embedded tissue specimens (M. Le Calve, et al., manuscript in preparation). The study was performed as previously described, ${ }^{29,39}$ and the molecular biology team was blinded to the clinical data and follow up. Individual samples embedded in paraffin blocks were examined histologically, and blocks predominantly composed of neoplastic cells were selected. Hematoxylin and eosin-stained slides served as a guide for the fluorescence microscopic study that was performed on interphase nuclei of 5- $\mu \mathrm{m}$ thick paraffin sections, and was performed according to the Vysis procedure (Vysis, Inc., Des Plaines, IL). The DNA probes were developed from different bacterial artificial chromosome clones specific to 1p36, 19q13, 1q23, and 19p23. Plasmid DNA extraction was performed according to the Qiagen protocol (Plasmid Midi Kit; Qiagen, Valencia, CA). Probes were directly labeled with either spectrum red (Vysis, Inc.) for 1p36 and 19q13 or spectrum green (Vysis, Inc.) for 1q23 and 19p23 by using the Nick Translation Kit (Vysis, Inc.). Probes were tested on normal metaphases for their specificity before being applied to paraffin sections, and the technique was first tested on 12 normal samples from our nervous tissue database.

For each specific chromosome probe, hybridization signals were scored from a minimum of 200 nonoverlapping nuclei. Counting was only effective when the nuclei showed clear signals and all fluorescent dots showed similar intensity. Deletions were defined as samples demonstrating one signal over more than $50 \%$ of the nuclei. Polysomia was confirmed on either chromosome when more than $10 \%$ of nuclei exhibited three or more signals for one or both probes.

\section{Patient Population}

Of 145 newly diagnosed oligodendrogliomas, 33 tumors with neoplastic astrocytes or an astrocytic component of more than $20 \%$ of the total cells were classified as oligoastrocytomas and were excluded from the study. Twenty-five cases were excluded because the diagnosis was based on stereotactic biopsy sampling, which may not necessarily sample a representative specimen of the lesion. The charts of the remaining 87 patients with supratentorial oligodendroglioma were reviewed. Clinical data systematically assessed included patient age, sex, presenting symptom, duration of symptoms, neurological examination at admission, and tumor location.

All patients underwent either CT (with and without contrast agent) and/or MR imaging (with and without gadolinium) studies performed before surgery and repeated within 72 hours after surgery.

All patients underwent subtotal or gross-total resection. The neuroimaging studies were reanalyzed when available (69 patients) to confirm the presence or absence of contrast enhancement of the tumor and the quality of the resection; otherwise the operative record and data obtained by the neurooncology staff were consulted.

Total excision was performed if there was no residual enhancement for the high-grade nor hypodensity or hyposignal for the lower-grade lesions; otherwise subtotal excision was performed.

Survival time was measured from the date of surgery until death or the last clinical evaluation before January 12 , 2004. The mean follow-up period from surgery was $61 \pm$ 40 months (range $3-160$ months), and no patients were lost to follow up. In 19 patients the date of death was obtained from the local record office. Neither quality of life nor the Karnofsky Performance Scale score was evaluated. No patient experienced leptomeningeal dissemination or distant metastasis.

All patients were treated surgically, and all patients included in the study survived the procedure. Adjuvant therapy was not performed in 21 patients $(24 \%) ; 36(41 \%)$ received radiotherapy or chemotherapy, 29 (33\%) only radiotherapy, and one patient only chemotherapy.

The median physical radiation dose was 54 Gy (range 45-60 Gy) with no dose difference related to age or sex. The number of chemotherapy-associated "cures" variable, with a mean of five cures (range three-15). A combination regimen of procarbazine, lomustine, and vincristine was the most commonly used.

\section{Statistical Analysis}

The statistical values were computed using available software (SPSS Version 12.0 for Windows; SPSS, Inc., Chicago, IL). Correlations of categorical variables were investigated using the Pearson correlation test. Bilateral associations showing statistical significance at a probability level of 0.05 were considered in our analysis. 
The survival analysis first consisted of computing Kaplan-Meier survival curves to estimate the mean and median survival for each parameter. The survival curves were compared using univariate Cox regression analysis after checking for the proportionality of hazard and crude hazard ratios with their 95\% CIs and by using both the generalized Wilcoxon and the log-rank (Mantel-Cox) tests, with a probability value less than 0.05 considered statistically significant.

Time to progression was analyzed using the KaplanMeier model, and patients with or without local progression were considered for analysis of this variable, the event being recurrence.

A multivariate Cox analysis was performed using Wald criteria and both forward and backward regression.

\section{RESULTS}

The data for our study population are summarized in Table 1.

\section{Age and Sex Distribution}

The 87 patients included 48 women (55\%) and 39 men (45\%). The overall mean age at presentation was $41 \pm 15$ years (range 5-73 years). The women were older (mean $45 \pm 16$ years [range 11-73 years]) than the men (mean $35 \pm 13$ years [range 5-67 years]), and this difference was statistically significant $(\mathrm{p}=0.006)$.

The chromosomal anomaly distribution was homogeneous with regard to sex and age.

\section{Clinical Features}

Seizures were the most frequent presenting symptom (66\%) compared with other presenting symptoms, including signs of intracranial hypertension, neurological deficit, and mental deterioration. Seizure remained the most frequent presenting symptom, even when adjusted to chromosomal findings, and was most often linked to a normal neurological examination (50 of 59 cases), whereas other presenting symptoms were usually linked to an abnormal neurological examination (21 of 28 cases).

The mean duration of symptoms before tumor detection was 16.5 months (range 1 day-132 months) when seizures appeared at onset and 8 months (range 1-102 months) for other presenting symptoms. The interval between onset and diagnosis was longer in patients younger than 50 years (16 months) than in those older than 51 years ( 7 months). Adjusted to chromosomal anomalies, the mean duration of symptoms was shorter in patients with polysomia (5 months) than in patients with $1 \mathrm{p}$ and $19 \mathrm{q}$ deletions (15 months) or without such deletions (16 months).

The most commonly involved anatomical site was the frontal lobe (59\%), with a significant statistical correlation between location and first symptom. In 40 frontal locations (74\%) the presenting symptom was epilepsy. There was no correlation between chromosomal anomalies and onset, neurological examination, or location.

\section{Neuroimaging Features}

Contrast enhancement on neuroimaging studies correlated with both presenting symptoms and findings at the clinical examination. Enhancing lesions were most frequent in patients who presented with symptoms other than seizure. In patients with epilepsy as the first symptom, the rates of enhancing and nonenhancing lesions were similar regardless of age.

Of 28 patients whose neurological examination demonstrated abnormal findings, 26 (93\%) had enhancing lesions, and there was no difference when the findings of the neurological examination were normal (28 of 59 patients had nonenhancing lesions). No correlation existed between chromosomal anomalies and neuroimaging features.

\section{Molecular Analysis}

The FISH assay revealed three categories of patients: the first group with a $1 p$ and/or $19 q$ deletion, the second group with no $1 \mathrm{p}$ or $19 \mathrm{q}$ deletion, and the third group in which polysomia was observed.

\section{Histological Features}

Of the clinicopathological correlations observed, we focused on the link between vascular proliferation and neuroimaging enhancement. This correlation was positive in $78 \%$ of cases. In 54 cases with histological vascular proliferation imaging enhancement was evident, and in 14 cases a lack of vascular proliferation and imaging enhancement was observed. In $22 \%$ of cases, the correlation was negative. In 16 patients with vascular proliferation no imaging enhancement was demonstrated and three tumors that enhanced on neuroimages had no vascular proliferation. None of the histological parameters was linked to specific chromosomal anomalies.

Thirty-two tumors $(36 \%)$ were histologically classified as low-grade oligodendroglioma (WHO Grade II), and the remaining 53 tumors (54\%) represented anaplastic tumors (Grade III). When tumors were graded by the St. Anne classification, $14(16 \%)$ were classified as Grade A and 73 $(84 \%)$ as Grade B. The discrepancy between the two grading systems is due to a misclassification of some intermediate grades representing benign histological features and imaging enhancement. There was no relationship between the WHO or St. Anne grade and the chromosomal findings.

\section{Patient Survival}

Univariate Analysis. Table 2 shows the survival time according to clinicopathological features and treatment modalities. Thirty-two deaths (37\%) were recorded (nine patients with Grade II and 23 with Grade III lesions compared with five patients with Grade A and 27 with Grade B lesions).

The median follow-up period was 53 months. The mean overall survival was $107 \pm 40$ months (range 3-160 months). The overall median survival was not reached.

Men survived longer than women; however, after adjustment for age there was no difference in survival between the two sexes. Age was of prognostic value. Seizures were of good prognostic value compared with other presenting symptoms. Findings of the neurological examination were of prognostic value. Patients with normal neurological findings survived longer than patients with neurological deficits. Patients with enhancing lesions had worse outcomes than those with nonenhancing lesions. Tumor location alone was not predictive of survival. Of the histological fea- 
A. Hamlat, et al.

TABLE 1

Clinical and pathological findings, molecular genetic characteristics, and outcomes in the patient population*

\begin{tabular}{|c|c|c|c|c|c|c|c|c|c|c|c|}
\hline \multirow[b]{3}{*}{ Variables } & \multirow{3}{*}{$\begin{array}{l}\text { No. of } \\
\text { Patients }\end{array}$} & \multirow{2}{*}{\multicolumn{3}{|c|}{ Age (yrs) }} & \multirow{2}{*}{\multicolumn{2}{|c|}{ Sex }} & \multirow{2}{*}{\multicolumn{2}{|c|}{$\mathrm{CT} / \mathrm{MRI}$}} & \multicolumn{3}{|c|}{ Chromosomal Anomalies } \\
\hline & & & & & & & & & \multirow{2}{*}{$\begin{array}{c}1 \mathrm{p} / 19 \mathrm{q} \\
\text { Deletion }\end{array}$} & \multirow{2}{*}{$\begin{array}{c}\text { No } \\
\text { Deletion }\end{array}$} & \multirow[b]{2}{*}{ Polysomia } \\
\hline & & $<25$ & $26-50$ & $\geq 51$ & M & $\mathrm{F}$ & $\mathrm{NE}$ & $\mathrm{E}$ & & & \\
\hline age $0-25$ yrs & 13 & & & & 8 & 5 & 7 & 6 & 5 & 7 & 1 \\
\hline age $26-50$ yrs & 51 & & & & 26 & 25 & 19 & 32 & 34 & 5 & 12 \\
\hline age $\geq 51$ yrs & 23 & & & & 5 & 18 & 4 & 19 & 11 & 6 & 6 \\
\hline seizure & 57 & 10 & 37 & 10 & 29 & 28 & 26 & 31 & 35 & 11 & 11 \\
\hline other presenting symptoms & 30 & 3 & 14 & 13 & 10 & 20 & 4 & 26 & 15 & 7 & 8 \\
\hline \multicolumn{12}{|l|}{ examination } \\
\hline normal & 59 & 13 & 38 & 8 & 29 & 30 & 28 & 31 & 35 & 10 & 14 \\
\hline abnormal & 28 & 0 & 13 & 15 & 10 & 18 & 2 & 26 & 15 & 8 & 5 \\
\hline \multicolumn{12}{|l|}{ location of tumor } \\
\hline frontal & 51 & 8 & 30 & 13 & 24 & 27 & 19 & 32 & 27 & 12 & 12 \\
\hline weak-moderate & 47 & 9 & 29 & 9 & 22 & 25 & 25 & 22 & 22 & 11 & 14 \\
\hline intense & 40 & 4 & 22 & 14 & 17 & 23 & 5 & 35 & 28 & 7 & 5 \\
\hline absent & 64 & 9 & 41 & 14 & 29 & 25 & 30 & 34 & 38 & 12 & 14 \\
\hline present & 23 & 4 & 10 & 9 & 10 & 13 & 0 & 23 & 12 & 6 & 5 \\
\hline \multicolumn{12}{|l|}{ vascular proliferation } \\
\hline absent & 17 & 4 & 10 & 3 & 7 & 10 & 14 & 3 & 7 & 4 & 6 \\
\hline present & 70 & 9 & 41 & 20 & 32 & 38 & 16 & 54 & 43 & 14 & 13 \\
\hline \multicolumn{12}{|l|}{ mitoses } \\
\hline$<5$ cells/hpf & 64 & 12 & 40 & 12 & 32 & 32 & 29 & 35 & 38 & 12 & 14 \\
\hline$>5$ cells $/ \mathrm{hpf}$ & 23 & 1 & 11 & 11 & 7 & 16 & 1 & 22 & 12 & 6 & 5 \\
\hline \multicolumn{12}{|l|}{ grading } \\
\hline WHO Grade II & 32 & 7 & 21 & 4 & 15 & 17 & 21 & 11 & 13 & 7 & 12 \\
\hline WHO Grade III & 55 & 6 & 30 & 19 & 24 & 31 & 9 & 46 & 37 & 11 & 7 \\
\hline \multicolumn{12}{|l|}{ outcome } \\
\hline dead & 32 & 0 & 14 & 18 & 8 & 24 & 6 & 26 & 10 & 8 & 14 \\
\hline alive & 55 & 13 & 37 & 5 & 31 & 24 & 24 & 31 & 40 & 10 & 5 \\
\hline no recurrences & 50 & 10 & 26 & 14 & 28 & 22 & 17 & 33 & 33 & 12 & 5 \\
\hline recurrences & 37 & 3 & 25 & 9 & 11 & 26 & 13 & 24 & 17 & 6 & 14 \\
\hline
\end{tabular}

$* \mathrm{E}=$ enhanced $; \mathrm{NE}=$ nonenhanced

$\dagger$ One patient had only surgery and chemotherapy and was not included.

tures, absence of necrosis and mitoses were related to survival and had statistical significance. Tumor grade, as determined using either the WHO or St. Anne grading system, had no statistically significant effect on patient survival; however, WHO grading was of prognostic value with regard to the chromosomal anomaly (Fig. 1).

A review of the records did not reveal why some patients underwent postoperative radiotherapy and others did not, nor the timing of chemotherapy. This therefore raises the question of whether selection bias occurred. The distribution of treatment modalities according to patient age and clinical examination was inconsistent. The median ages were 30,40 , and 50 years for the groups treated by surgery, surgery plus radiotherapy and chemotherapy, and surgery plus radiotherapy, respectively. In most patients who underwent surgery and radiotherapy the clinical examination yielded abnormal findings in contrast to those patients treated by surgery alone, in whom the findings were normal. We therefore did not conduct a comparative study of the different treatment modalities.

The extent of resection (mean survival 109 months for total excision compared with 101 months for subtotal excision), even adjusted to chromosomal anomalies, was not of prognostic value. The overall mean survival period for the 
TABLE 2

Survival period according to clinical parameters*

\begin{tabular}{|c|c|c|c|c|c|c|c|}
\hline Parameter & $\begin{array}{l}\text { No. of } \\
\text { Patients }\end{array}$ & Groups & \multicolumn{5}{|c|}{ Overall Survival (mos) } \\
\hline \multirow[t]{2}{*}{ age (yrs) } & 13 & $0-25$ & censored & & - & - & $0.0000 \dagger$ \\
\hline & 23 & $>50$ & $34 \pm 6$ & $22-47$ & $20 \pm 9$ & $3-37$ & \\
\hline \multirow[t]{2}{*}{ sex } & 48 & female & $86 \pm 11$ & $65-107$ & $82 \pm 29$ & $26-138$ & $0.0025 \dagger$ \\
\hline & 39 & male & $124 \pm 8$ & $108-141$ & - & - & \\
\hline \multirow[t]{2}{*}{ neurological examination } & 59 & normal & $120 \pm 7$ & $106-134$ & - & - & $0.0000 \dagger$ \\
\hline & 28 & abnormal & $68 \pm 13$ & $42-93$ & $27 \pm 9$ & $10-44$ & \\
\hline \multirow[t]{2}{*}{ neuroimaging enhancement } & 57 & present & $95 \pm 9$ & $77-113$ & 93 & & $0.0231 \dagger$ \\
\hline & 30 & absent & $124 \pm 10$ & $105-142$ & & & \\
\hline \multirow[t]{2}{*}{ tumor location } & 51 & frontal & $114 \pm 9$ & $96-132$ & - & - & 0.2553 \\
\hline & 36 & nonfrontal & $80 \pm 9$ & $63-98$ & - & - & \\
\hline mitoses & 64 & $<5 / \mathrm{hpf}$ & $120 \pm 8$ & $104-135$ & - & - & $0.0008 \dagger$ \\
\hline \multirow[t]{2}{*}{ WHO Grade } & 32 & II & $114 \pm 10$ & $94-134$ & - & - & 0.1799 \\
\hline & 55 & III & $100 \pm 9$ & $82-119$ & - & - & \\
\hline \multirow[t]{2}{*}{ excision } & 49 & total & $109 \pm 10$ & $90-128$ & - & - & 0.9564 \\
\hline & 38 & subtotal & $101 \pm 10$ & $81-121$ & - & - & \\
\hline \multirow[t]{3}{*}{ treatment modalities } & 87 & overall survival & $107 \pm 7$ & $93-122$ & - & - & \\
\hline & 66 & surgery \& radiotherapy & $94 \pm 9$ & $77-111$ & 93 & & \\
\hline & 37 & surgery \& chemotherapy & $118 \pm 11$ & $97-139$ & - & - & \\
\hline \multirow[t]{3}{*}{ chromosomal anomalies } & 50 & $1 \mathrm{p}-19 \mathrm{q}$ deletion & $130 \pm 9$ & $113-146$ & - & - & $0.0001 \dagger$ \\
\hline & 18 & no $1 \mathrm{p}-19 \mathrm{q}$ deletion & $83 \pm 14$ & $56-109$ & - & - & \\
\hline & 19 & polysomia & $51 \pm 10$ & $31-71$ & - & - & \\
\hline \multirow{3}{*}{ time to disease progression } & 87 & overall & $92 \pm 8$ & $76-108$ & 72 & & \\
\hline & 37 & $\mathrm{w} /$ chemotherapy & $115 \pm 11$ & $93-137$ & - & - & \\
\hline & 66 & $\mathrm{w} /$ radiotherapy & $101 \pm 9$ & $82-119$ & - & - & \\
\hline
\end{tabular}

$* \mathrm{SE}=$ standard error; $-=$ not reached or not applicable.

$\dagger$ Statistically significant.

entire group was 107 months. The overall survival adjusted to radiotherapy or chemotherapy indicated that chemotherapy improved overall survival, but radiotherapy did not.

Survival adjusted to chromosomal anomalies was 130 months for both $1 \mathrm{p}$ and $19 \mathrm{q}$ deletions (Fig. 2 left). Survival adjusted both to chromosomal anomalies and radiotherapy showed no benefit from radiotherapy (Fig. 2 center), whereas chemotherapy did improve survival (Fig. 2 right).

In our paper, we use the term local progression rather than recurrence. Even when macroscopic resection is total, complete histological removal is seldom achieved due to the infiltrative growth pattern of oligodendroglioma. All tumor progression occurring in the immediate vicinity of the primary tumor indicated a poor prognosis.

The overall mean survival period of patients with no local progression was 128 months compared with 77 months in those with local progression $(p=0.0021)$. The rate of local progression stratified to chromosomal anomalies was $33 \%$ in the single chromosome deletion and no-deletion groups compared with $74 \%$ in the polysomic group $(\mathrm{p}=$ $0.008)$. The death rate was $20 \%$ for $1 p-19 q$ deletion, $44 \%$ for no $1 \mathrm{p}-19 \mathrm{q}$ deletion, and $73 \%$ for polysomia $(\mathrm{p}=0.005)$.

The overall mean time to local progression was 92 months, and there was no difference between total excision (mean 93 months) and subtotal excision (mean 87 months). Radiotherapy improved the overall mean time to progression (101 months) although not to a statistically significant extent, whereas chemotherapy did so significantly (mean 115 months). The mean time to progression adjusted to chromosomal anomalies was 107 months for patients with 1p-19q deletions (Fig. 3 left). Radiotherapy, adjusted to chromosomal anomalies in the $1 \mathrm{p}-19 \mathrm{q}$ deletion group, significantly improved time to progression (mean 122 months; Fig. 3 center). Chemotherapy, adjusted to chromosomal anomalies, also significantly improved the time to progression in patients with 1p-19q deletions (mean 145 months; Fig. 3 right).

\section{Statistical Analysis}

The predictive power of chromosomal anomalies was further assessed by logistic regression. The Cox regression model showed that only age $\left(\mathrm{p}<10^{-5}\right)(\mathrm{OR} 1.026,95 \% \mathrm{CI}$ 1.049-1.074) and chromosomal anomalies for no deletion $(\mathrm{p}<0.002$; OR 3.5, 95\% CI 4.3-9.2) and for polysomia with $1 \mathrm{p}-19 \mathrm{q}$ deletion as a reference group (OR 7.4, 95\% CI 3-13.7), were independently linked with survival. 


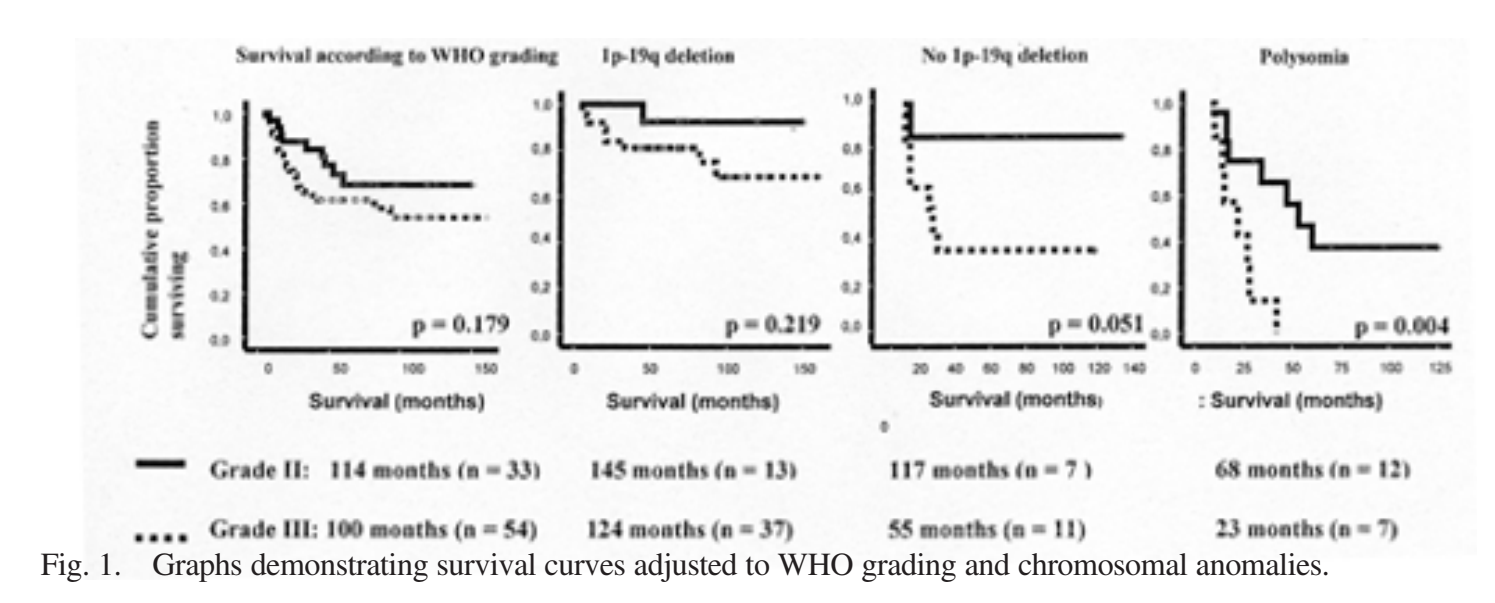

For time to progression, none of the prognostic factors analyzed emerged as independent prognostic factors.

\section{DISCUSSION}

Oligodendrogliomas constitute approximately 2 to $9 \%$ of all primary brain tumors ${ }^{25,30,43}$ and 25 to $33 \%$ of all glial tumors. ${ }^{10,14}$ In the US, the annual incidence of all oligodendroglioma was reported to be 0.3 per 100,000 individuals in $1995 .{ }^{8}$ It is well known that several investigators have come to different conclusions when clinical, pathological, and treatment modalities have been correlated with survival. This is possibly due to the lack of biological markers for accurate diagnosis, ${ }^{13,34}$ absence of an accepted grading system or unambiguous malignancy criteria, and use of different therapeutic modalities. ${ }^{14,34,37}$ In a single-center populationbased retrospective-prospective study, we investigated and compared chromosomal assay with clinical and histopathological features and treatment modalities in 87 oligodendrogliomas. Univariate and multivariate analyses yielded disparate prognostic factors. Some of these merit emphasis.

\section{Clinical Data}

The overall patient population in this series is similar to other oligodendroglioma case series ${ }^{7,10,11,23,24,26,28,30,37,43}$ except that in this study there were more women than men. ${ }^{38,47}$ In most studies, age was found to affect survival using either univariate ${ }^{7,16,23,24,26,37,38,49}$ or multivariate analysis. ${ }^{6,25,33,51}$ It has been shown that the observed survival distribution was significantly different from the corresponding expected agesex matched survival curves of a normal reference population. ${ }^{37}$ In our data, age emerges as an independent prognostic factor.

The effect of age may distort the effect of sex on survival because the median age of the women in this series was 10 years older than that of the men. When sex is adjusted to age, it has no statistical value. Therefore, the sex difference in survival is only a statistical bias attributable to the older age of women.

In most series, seizure was the most common presenting symptom, ranging in incidence from 35 to $91 \%$ of patients; $7,10,11,28,30,33,38,43$ it was reported as a prognostic factor. ${ }^{10,25,30,45}$ There was a strong correlation between the mode of presentation and clinical examination. Seizures are most often found in patients whose neurological examination was normal, in both younger and older patients in contrast to abnormal neurological examination which is most often associated with other presenting symptoms and older age. Seizure is a statistically significant prognostic factor independent of chromosomal anomalies.

Furthermore, clinical status was also reported to be statistically significant in relation to survival. $7,9,21,26,27,30,43,45,49$ Patients without neurological deficits fared better. Moreover, permanent neurological deficits probably represent a factor of comorbidity in older patients. Our data confirm that the frontal lobe is the principal tumor location, $7,10,11,28,30$, $33,37,38,43,49,51,52$ although this has neither prognostic significance $^{7,9,11,16,22,24}$ nor is it linked to chromosomal anomalies, as reported previously. ${ }^{52}$

\section{Radiological Features}

Although imaging studies provide valuable information, none has proven specific for oligodendroglial differentiation. Of the imaging features, radiological enhancement was closely related to vascular proliferation and both were considered criteria for diagnosis of malignancy and assessment for prognosis. . $25,26,37,46$ In our series, enhancement emerges as a prognostic factor, and its clinical significance merits discussion. Some patients underwent CT scanning and others MR imaging. Thus the evaluation was not uniform. Furthermore, in $22 \%$ of cases there was no relationship between enhancement and malignancy (some lowgrade oligodendrogliomas showed enhancement and some anaplastic ones did not). Nevertheless, as reported previously, ${ }^{10} 93 \%$ (26 of 28) of our patients with neurological deficits had enhancing tumors. Thus, from our own experience, and as demonstrated previously, ${ }^{6,15}$ we only attribute a malignant value to enhancement if necrosis is also present (ring enhancement).

\section{Pathological Features}

Numerous attempts have been made to identify characteristics of prognostic significance, and the literature has presented the conflicting views of authors regarding the influence of individual pathological features on survival. Therefore, immunohistological features linked to a poor outcome included necrosis, high mitotic activity, increased cellularity, nuclear atypia, cellular pleomorphism, micro- 
vascular proliferation, and the presence of vascular endothelial growth factor, MIB1, and other biological markers. ${ }^{6,9}$, $11,13,14,21,22,24,27,30,33,41,46$ In our series, we could only relate survival to mitosis and necrosis by using univariate analysis.

Recent investigators have demonstrated that these basic pathological features, which are also used for grading oligodendrogliomas, are in themselves unreliable $e^{14}$ and have different intrinsic impact on survival. Their influence is highly variable in the previous series as demonstrated by the variable results of the series including patients with the same pathological features. $7,9,11,16,23,25,26,30,37,43,46,47,51$

The lack of correlation between histopathological and molecular analyses (as shown in Table 1) suggests that the histological group of oligodendroglioma defined by the two pathological grading systems is genetically heterogeneous. This may explain the varying results observed in previous studies in which histopathological features were the sole prognostic factors. Therefore, genetic analysis may be a particularly useful tool for classifying oligodendroglioma. ${ }^{14,34,48}$ Molecular genetic assay does not foretell the end of traditional histopathological analysis, however, but rather it will enhance its interpretation. ${ }^{48}$

\section{Molecular Assay}

Fluorescence in situ hybridization may not detect genetic events that do not result in a change in copy number, specifically loss of one allele followed by duplication of the remaining allele. ${ }^{31}$ Some knowledge of the abnormality is necessary to select the appropriate probes. ${ }^{19}$ Nonetheless, as reported in anaplastic oligodendroglioma, ${ }^{15}$ our series demonstrates that oligodendroglioma may be divided into the following three distinct therapeutic and prognostic subgroups based on molecular biology: 1) oligodendroglioma showing $1 \mathrm{p}-19 \mathrm{q}$ deletion with a favorable prognosis; 2 ) polysomic oligodendroglioma characterized by a lack of meaningful response to both radiotherapy and chemotherapy with a particularly ominous prognosis and a high rate of recurrence and death; and 3) oligodendroglioma with no $1 p-19 q$ deletion, which are intermediate between the first two molecular subtypes depending on histological grading.

The incidence of $1 \mathrm{p}-19 \mathrm{q}$ deletion does not differ in specimens from primary and metastatic lesions, ${ }^{40}$ and it has been shown that malignant progression is associated with the loss of $10 \mathrm{q},{ }^{15,17,35,36} 9 \mathrm{p}, 3,4$ and the accumulation of multiple genetic anomalies. ${ }^{3,4,17,35,36}$

A subset of 196 genes are differentially expressed between WHO Grades II and III, and 209 genes are differentially expressed between oligodendroglioma with and without $1 \mathrm{p}$ loss. ${ }^{34}$ In addition, detailed analysis of the messenger RNA expression of oligodendroglioma has shown a preponderance of larger-sized transcripts for several genes that may represent a fundamental difference in oligodendroglioma tumorigenesis..$^{50}$

We can speculate that the entire group, and specifically those patients with no $1 \mathrm{p}-19 \mathrm{q}$ deletion and polysomia, may have other chromosomal anomalies. Thus, the distinct survival rate and response to treatment observed within the molecular subgroups may be improved by identification of new basic molecular differences in the future.

\section{Treatment Modalities}

The issue of how to treat oligodendroglioma adequately is still a matter of debate. $1,2,7,8,12,16,32,47,51$ Authors of some reports have indicated that a more complete resection of tumor is associated with increased survival. ${ }^{7,11,16,21,27,37}$ Our data have not confirmed this, as the survival period was not affected by residual tumor. We did not compare treatment modalities because of statistical bias. Nonetheless, from the review of the patients treated by excision alone, we can infer that, in younger patients with an asymptomatic lowgrade oligodendroglioma and $1 \mathrm{p}-19 \mathrm{q}$ deletion, total resection is adequate and adjuvant treatment should be deferred until the tumor progresses because all such patients are still alive.

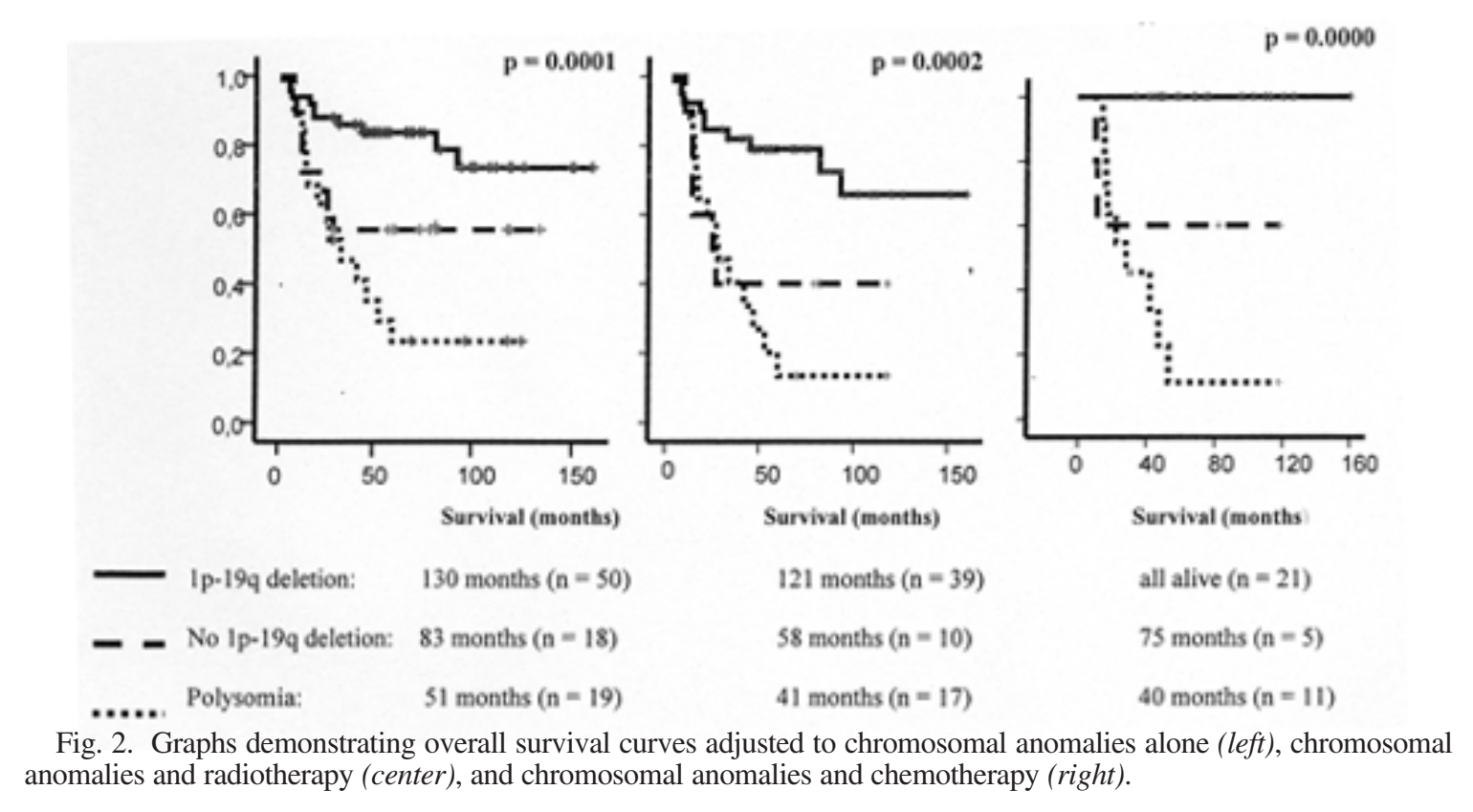




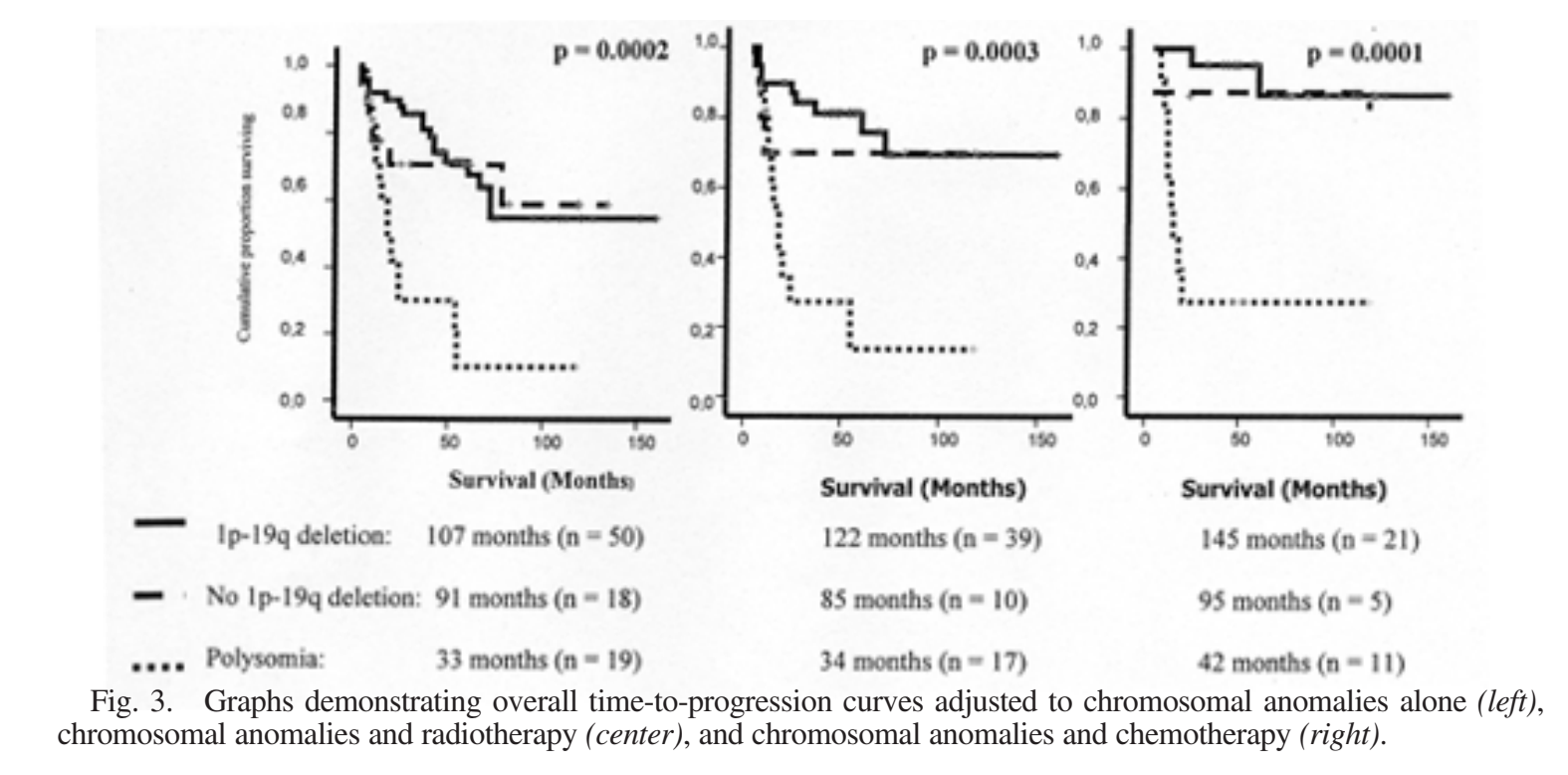

The role of radiotherapy has been questioned, and there is no demonstration in the literature that radiotherapy is always effective in treating oligodendroglioma. $7111,14,27,37,38,43$ Findings from the present series suggest that there is no beneficial effect of radiotherapy alone on survival, even when age, clinical data, histological grading, and type of surgery are considered; however, radiotherapy prolongs the overall time to progression.

Because the timing of radiotherapy in the postoperative setting is unclear, it could be argued that any survival benefit derived from radiotherapy in the immediate postoperative period could be biased by its effect on local progression.

Our data confirm that chromosomal deletions of $1 p-19 q$ are associated with better survival and response to chemotherapy. The survival curves show a plateau in patients with $1 p-19 q$ deletions treated by adjuvant chemotherapy, which also prolongs the time to progression; however, radiation therapy and chemotherapy improved neither survival nor the free time to progression in patients who did not have $1 p-19 q$ deletion and polysomia, although the patient numbers were limited.

In the literature, the response rate of oligodendroglioma to chemotherapy ranges from 60 to $100 \%, 4,6,12,14,15,32$ and the response rate is similar according to histological grade., ${ }^{8,21}$

\section{CONCLUSIONS}

This study reveals three molecular subgroups of oligodendroglioma which have clinically different outcomes. Although combined therapy of surgery, radiotherapy, and chemotherapy is currently considered one of the standard treatments of oligodendrogliomas, a comparative prospective randomized study between surgery plus radiotherapy and surgery plus chemotherapy seems necessary for evaluating oligodendrogliomas with $1 \mathrm{p}-19 \mathrm{q}$ deletion and those without these chromosomal anomalies.

\section{Acknowledgments}

We are grateful to Prof. Abed El-Ghalem for his help and advice,
Ms. T. Westcott for correcting the English, and the Brain Neuro-Oncology staff for their support (Mahmoudreza Adn, Gilles Brassier, Beatrice Carsin-Nicol, Christine Edan, Joelle Egreteau, Isabelle LeCouillard, Thierry Lesimple, Xavier Morandi, Laurent Riffaud).

\section{Dedication}

This article is dedicated to the patients who died of oligodendroglioma.

\section{References}

1. Abrey LE, Childs BH, Paleologos N, et al: High-dose chemotherapy with stem cell rescue as initial therapy for anaplastic oligodendroglioma. J Neurooncol 65:127-134, 2003

2. Bélanger K, MacDonald D, Cairncross G, et al: A phase II study of topotecan in patients with anplastic oligodendroglioma or anaplastic mixed oligoastrocytoma. Invest New Drugs 21: 473-480, 2003

3. Bigner SH, Matthews MR, Rasheed BKA, et al: Molecular genetic aspects of oligodendrogliomas including analysis by comparative genomic hybridization. Am J Pathol 155:375-386, 1999

4. Bourdon V, Plessis G, Chapon F, et al: Chromosome imbalances in oligodendroglial tumors detected by comparative genomic hybridization. Ann Genet 47:105-111, 2004

5. Cairncross JG, Ueki K, Zlatescu MC, et al: Specific genetic predictors of chemotherapeutic response and survival in patients with anaplastic oligodendrogliomas. J Natl Cancer Inst 90: 1473-1479, 1998

6. Cavalla P, Piva R, Bortolotto S, et al: p27/kip1 expression in oligodendrogliomas and its possible prognostic role. Acta Neuropathol 98:629-634, 1999

7. Celli P, Nofrone I, Palma L, et al: Cerebral oligodendroglioma: prognostic factors and life history. Neurosurgery 35: 1018-1025, 1994

8. Chahlavi A, Kanner A, Peerboom D, et al: Impact of chromosome 1p status in response of oligodendroglioma to temozolomide: preliminary results. J Neurooncol 61:267-273, 2003

9. Daumas-Duport C, Tucker ML, Kolles H, et al: Oligodendrogliomas. Part II: A new grading system based on morphological and imaging criteria. J Neurooncol 34:61-78, 1997

10. Daumas-Duport C, Varlet P, Tucker ML, et al: Oligodendrogliomas. Part I: patterns of growth, histological diagnosis, clinical and imaging correlations: a study of 153 cases. J Neurooncol 34: 37-59, 1997 
11. Deghani F, Schachenmayr W, Laun A, et al: Prognostic implication of histopathological, immunohistochemical and clinical features of oligodendrogliomas: a study of 89 cases. Acta Neuropathol (Berl) 95:493-504, 1998

12. Diabira S, Rousselet MC, Gamelin E, et al: PCV chemotherapy for oligodendroglioma: response analyzed on T2 weightedMRI. J Neurooncol 55:45-50, 2001

13. Engelhard HH, Stelea A, Cochran EJ: Oligodendroglioma: pathology and molecular biology. Surg Neurol 58:111-117, 2002

14. Fortin D, Cairncross GJ, Hammond RR: Oligodendroglioma: an appraisal of recent data pertaining to diagnosis and treatment. Neurosurgery 45:1279-1291, 1999

15. Ino Y, Betensky RA, Zlatescu MC, et al: Molecular subtypes of anaplastic oligodendroglioma: implications for patient management at diagnosis. Clin Cancer Res 7:839-845, 2001

16. Jeremic B, Shibamoto Y, Grujicic D, et al: Combined treatment modality for anplastic oligodendroglioma: a phase II study. J Neuro-Oncol 43:179-185, 1999

17. Jeuken JWM, Sprenger SHE, Vermeer H, et al: Chromosomal imbalances in primary oligodendroglial tumors and their recurrences: clues about malignant progression detected using comparative genomic hybridization. J Neurosurg 96:559-564, 2002

18. Johnson MD, Vnencak-Jones CL, Toms SA, et al: Allelic losses in oligodendroglial and oligodendrglioma-like neoplasms: analysis using microsatellite repeats and polymerase chain reaction. Arch Pathol Lab Med 127:1573-1579, 2003

19. Kearney L: Molecular cytogenetics. Best Pract Res Clin Haematol 14:645-669, 2001

20. Kleihues P, Louis DN, Scheithauer BW, et al: The WHO classification of tumors of the nervous system. J Neuropathol Exp Neurol 61:215-229, 2002

21. Korshunov A, Golanov A: The prognostic significance of vascular endothelial growth factor (VEGF C-1) immunoexpression in oligodendroglioma. An analysis of 91 cases. J Neurooncol 48:13-19, 2000

22. Kros JM, Hop WC, Godschalk JJ, et al: Prognostic value of the proliferation-related antigen $\mathrm{Ki}-67$ in oligodendrogliomas. Cancer 78:1107-1113, 1996

23. Kros JM, Pieterman H, van Eden CG, et al: Oligodendroglioma: the Rotterdam-Dijkzigt experience. Clinical study. Neurosurgery 34:959-966, 1994

24. Kros JM, Troost D, van Eden CG, et al: Oligodendroglioma. A comparison of two grading systems. Cancer 61:2251-2259, 1988

25. Lebrun C, Fontaine D, Ramaioli A, et al: Long term outcome of oligodendrogliomas. Neurology 62:1783-1787, 2004

26. Leonardi MA, Lumenta CB: Oligodendrogliomas in the CT/MR-Era. Acta Neurochir (Wien) 143:1195-1203, 2001

27. Lindegaard KF, Mork SJ, Eide GE, et al: Statistical analysis of clinicopathological features, radiotherapy, and survival in 170 cases of oligodendroglioma. J Neurosurg 67:224-230, 1987

28. Ludwig CL, Smith MT, Godfrey AD, et al: A clinicopathological study of 323 patients with oligodendrogliomas. Ann Neurol 19:15-21, 1986

29. Mark HF: Fluorescent in situ hybridization as an adjunct to conventional cytogenetics. Ann Clin Lab Sci 24:153-163, 1994

30. Mork SJ, Lindegaard KF, Halvorsen TB, et al: Oligodendroglioma: incidence and biological behavior in a defined population. $\mathbf{J}$ Neurosurg 63:881-889, 1985

31. Nigro JM, Takahashi MA, Ginzinger DG, et al: Detection of $1 \mathrm{p}$ and $19 q$ loss in oligodendroglioma by quantitative microsatellite analysis, a real-time quantitative polymerase chain reaction assay. Am J Pathol 158:1253-1262, 2001

32. Peterson K, Paleologos N, Forsyth P, et al: Salvage chemotherapy for oligodendroglioma. J Neurosurg 85:597-601, 1996

33. Prayson RA, Mohan DS, Song P, et al: Clinicopathologic study of forty-four histologically pure supratentorial oligodendrogliomas. Ann Diagn Pathol 4:218-227, 2000
34. Reifenberger G, Louis DN: Oligodendroglioma: toward molecular definitions in diagnostic neuro-oncology. J Neuropath Exp Neurol 62:111-126, 2003

35. Sanson M, Leuraud P, Aguirre-Cruz L, et al: Analysis of loss of chromosome 10q, DMBT1 homozygous deletions, and PTEN mutations in oligodendrogliomas. J Neurosurg 97:1397-1401, 2002

36. Sasaki H, Zlatescu MC, Betensky RA, et al: PTEN is a target of chromosome 10q loss in anaplastic oligodendrogliomas and PTEN alterations are associated with poor prognosis. Am J Pathol 159:359-367, 2001

37. Shaw EG, Scheithauer BW, O'Fallon JR, et al: Oligodendrogliomas: the Mayo Clinic experience. J Neurosurg 76:428-434, 1992

38. Shimizu KT, Tran LM, Mark RJ, et al: Management of oligodendrogliomas. Radiology 186:569-572, 1993

39. Smith JS, Alderete B, Minn Y, et al: Localization of common deletion regions on $1 \mathrm{p}$ and $19 \mathrm{q}$ in human gliomas and their association with histological subtype. Oncogene 18:4144-4152, 1999

40. Smith JS, Perry A, Borell TJ, et al: Alterations of chromosome arms $1 p$ and $19 q$ as predictors survival in oligodendrogliomas, astrocytomas and mixed oligoastrocytomas. J Clin Oncol 18: 636-645, 2000

41. Smith MT, Ludwig CL, Godfrey AD, et al: Grading oligodendrogliomas. Cancer 52:2107-2114, 1983

42. Staudt LM: Molecular diagnosis of the hematologic cancers $\mathbf{N}$ Engl J Med 348:1777-1785, 2003

43. Sun ZM, Genka S, Shitara N, et al: Factors possibly influencing the prognosis of oligodendroglioma. Neurosurgery 22:886-891, 1988

44. van den Bent M, Chinot OL, Cairncross JG. Recent developments in the molecular characterization and treatment of oligodendroglial tumors. Neuro-oncol 5:128-138, 2003

45. van den bent MJ, Looijenga LH, Langenberg $\mathrm{K}$, et al: Chromosomal anomalies in oligodendroglial tumors are correlated with clinical features. Cancer 97:1276-1284, 2003

46. Vaquero J, Zurita M, Coca S, et al: Prognostic significance of clinical and angiogenesis-related factors in low-grade oligodendrogliomas. Surg Neurol 54:229-234, 2000

47. Wallner KE, Gonzales M, Sheline GE: Treatment of oligodendrogliomas with or without postoperative irradiation. J Neurosurg 68:684-688, 1988

48. Watson MA, Perry A, Budhjara V, et al: Gene expression profiling with oligonucleotide microarrays distinguishes World Heath Organization grade of oligodendrogliomas. Cancer Res 61:1825-1829, 2001

49. Wilkinson IM, Anderson JR, Holmes AE: Oligodendroglioma: an analysis of 42 cases. J Neurol Neurosurg Psychiatry 50: 304-312, 1987

50. Xu X, Joh HD, Pin S, et al: Expression of multiple larger-sized transcripts for several genes in oligodendrogliomas: potential markers for glioma subtype. Cancer Lett 171:67-77, 2001

51. Yeh SA, Lee TC, Chen HJ, et al: Treatment outcomes and prognostic factors of patients with supratentorial low-grade oligodendroglioma. Int J Radiat Oncol Biol Phys 54:1405-1409, 2002

52. Zlatescu MC, TehraniYazdi R, Sasaki H, et al: Tumor location and growth pattern correlate with genetic signature in oligodendroglial neoplasms. Cancer Res 61:6713-6715, 2001

Manuscript received July 25, 2005.

Accepted in final form October 4, 2005.

Address reprint requests to: Abderrahmane Hamlat, M.D., Service de neurochirurgie, CHRU Pontchaillou, Rue Henri Le Guilloux, 35000 Rennes Cedex 2, France. email: hamlat.abd@wanadoo.fr. 